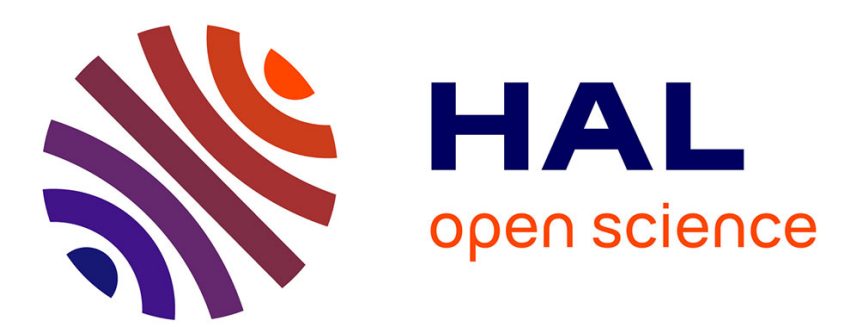

\title{
Modelling reflectometry diagnostics: finite-difference time-domain simulation of reflectometry in fusion plasmas
}

\author{
F da Silva, Stéphane Heuraux, E. Ricardo, A. F Silva, T. Ribeiro
}

\section{To cite this version:}

F da Silva, Stéphane Heuraux, E. Ricardo, A. F Silva, T. Ribeiro. Modelling reflectometry diagnostics: finite-difference time-domain simulation of reflectometry in fusion plasmas. Journal of Instrumentation, 2019, 14 (8), pp.C08003. 10.1088/1748-0221/14/08/C08003 . hal-02435292

\section{HAL Id: hal-02435292 \\ https://hal.science/hal-02435292}

Submitted on 10 Jan 2020

HAL is a multi-disciplinary open access archive for the deposit and dissemination of scientific research documents, whether they are published or not. The documents may come from teaching and research institutions in France or abroad, or from public or private research centers.
L'archive ouverte pluridisciplinaire HAL, est destinée au dépôt et à la diffusion de documents scientifiques de niveau recherche, publiés ou non, émanant des établissements d'enseignement et de recherche français ou étrangers, des laboratoires publics ou privés. 


\title{
Modelling reflectometry diagnostics: finite-difference time-domain simulation of reflectometry in fusion plasmas
}

\author{
F. da Silva, ${ }^{a}$ S. Heuraux,${ }^{b}$ E. Ricardo, ${ }^{a}$ A. Silva ${ }^{a}$ and T. Ribeiro ${ }^{c}$ \\ ${ }^{a}$ Instituto de Plasmas e Fusão Nuclear, Instituto Superior Técnico, Universidade de Lisboa, \\ 1049-001 Lisboa, Portugal \\ ${ }^{b}$ Institut Jean Lamour UMR 7198 CNRS-Université de Lorraine, \\ ARTEM BP 50840, F-54011 Nancy, France \\ ${ }^{c}$ Max-Planck-Institut für Plasmaphysik, \\ Garching D-85748, Germany \\ E-mail: tanatos@ipfn.tecnico.ulisboa.pt
}

ABSTRACT: Reflectometry simulations are particularly important since they allow to assess the measuring capabilities in experimental fusion devices and to predict the performance of future ones. We present a brief overview of reflectometry and introduce the family of REFMUL* codes for timedependent reflectometry simulation. REFMUL* codes are Finite Difference Time Domain (FDTD) that allow to set up synthetic diagnostics to assess the behaviour of reflectometry diagnostics. This is illustrated in the current manuscript using the example of the Plasma Position Reflectometers of DEMO.

KeYwords: Nuclear instruments and methods for hot plasma diagnostics; Simulation methods and programs; Plasma diagnostics - interferometry, spectroscopy and imaging 


\section{Contents}

1 Introduction 1

2 Microwave reflectometry 1

3 Why the need for complex simulation codes?

3.1 The REFMUL* code family 2

3.2 REFMULF and REFMUL3 geometry and equations 3

4 Examples of applications using REFMUL* codes 4

5 Conclusions 6

\section{Introduction}

Microwave reflectometry has become one of the most important techniques to diagnose the electronic density $n_{e}$ of fusion plasmas $[1,2]$. It has its origins in the techniques used to probe the ionosphere [3]. Reflectometry simulations are particularly important since they allow to assess the measuring capabilities of existing experimental systems and to predict the performance of future ones. We will introduce the reader to the family of REFMUL* codes. These time-dependent codes allow to set up synthetic diagnostics that not only consider the wave propagation in a given time-dependent plasma, but also take into account the system's location within the vacuum vessel, together with a characterisation of its access to the plasma (wave guides and antennas). We illustrate the use of reflectometry synthetic diagnostics in the assessment of the Plasma Position Reflectometers (PPR) of DEMO.

\section{Microwave reflectometry}

This diagnostic relies in the propagation of waves in a plasma. A probing signal $s_{\mathrm{src}}=A_{\mathrm{src}} \cos (\omega t)$ is launched through a plasma where it propagates until being reflected at a cutoff surface $x_{c}$, where the refraction index is zero $N\left(x_{c}\right)=0$. The received reflected signal $s_{\mathrm{rcv}}=A_{\mathrm{rcv}} \cos (\omega t+\varphi)$, differs from the emitted one in amplitude and phase. This phase shift $\varphi=\varphi_{\mu}+\varphi_{0}+\varphi_{p}$ results from different contributions: propagation in the microwave circuitry, $\varphi_{\mu}$; in a vacuum, $\varphi_{0}$; and in the plasma $\varphi_{p}$. A careful calibration of the system is done to determine $\varphi_{\mu}$ and a good approximation to $\varphi_{0}$ can be inferred. This leaves us with the phase difference $\varphi_{p}$, that reflects the propagation of the wave along a path described by a refractive index $N(\mathbf{r})$ and contains information on the electronic density $n_{e}$. The Altar-Appleton equation (aka Appleton-Hartree or Appleton-Lassen) describes the refractive index of a wave propagation in a cold magnetised plasma [4], where a magnetic field $\mathbf{B}_{\mathbf{0}}$ exists. Of interest to reflectometry is the particular case of propagation perpendicular to the external magnetic 
field $\mathbf{k} \perp \mathbf{B}_{\mathbf{0}}$ which gives two expressions. One for the ordinary mode (O-mode) with $N^{2}=1-X$ and another for the extraordinary mode (E- or X-mode) with $N^{2}=1-X(1-X) /\left(1-X-Y^{2}\right)$ [5]. Note the normalisation $X=\omega_{p}^{2} / \omega^{2}$ and $Y=\omega_{c} / \omega$, being $\omega_{p}, \omega_{c}$ and $\omega$ the plasma, the electron cyclotron and the wave angular frequencies, respectively. In the WKBJ framework ignoring the scattering effects, the O-mode describes a linear polarised wave with its electric field parallel to $\mathbf{B}_{\mathbf{0}}$ $\left(\mathbf{E} / / \mathbf{B}_{\mathbf{0}}\right)$ while the $\mathrm{X}$-mode describes an elliptical polarisation with the wave electric field parallel to $\mathbf{B}_{\mathbf{0}}\left(\mathbf{E} \perp \mathbf{B}_{\mathbf{0}}\right)$. For the O-mode, the phase due to plasma propagation $\varphi_{p}$ is a function of the wave frequency $f$ and the plasma electronic frequency $\omega_{p}, \varphi_{p}\left[f, N_{O}\left(n_{e}\right)\right]$ while for the X-mode also depends on $\mathbf{B}_{\mathbf{0}}, \varphi_{p}\left[f, N_{X}\left(n_{e}, B_{0}\right)\right]$.

\section{Why the need for complex simulation codes?}

From the previous introduction it would seem that the solid mathematical description of wave propagation in plasmas provides the necessary tools to interpret reflectometry experimental results obtained from experiments in fusion devices. Why all the investment in the development of complex simulation codes? It turns out that the analytic solutions describing propagation in plasmas must forcibly include some simplifications and the resulting ideal situation, such as the one depicted in figure 1 (left) exists, in fact, only in books as in [5]. Propagation in a thermonuclear plasma is far from trivial since the plasma is an extremely complex, inhomogeneous, non-stationary, anisotropic medium, where waves suffer the effects of turbulence, magnetohydrodynamics, Doppler shifts, absorption, diffusion, mode conversion, to name a few. Thus the need of a numerical full-wave treatment based on a simplified model which retains the main mechanisms. Several Computational ElectroMagnetic (CEM) techniques have been used to tackle this problem, being finite-difference time-domain (FDTD) [6] using Yee's scheme [7] the one most up to the job. As an example we point to figure 1 (right), where a demonstration of the impact of forward scattering on reflectometry is shown. We can observe a multitude of phenomena that shatters the coherence of the probing beam. In general, an analytical description of such effects is not possible, leaving numerical simulation as the only means to evaluate them.

\subsection{The REFMUL* code family}

The REFMUL* family includes REFMUL, a 2D O-mode simulation code [8], REFMULX/REFMULXp: 2D X-mode (serial/parallel) simulation codes [9] and two recent additions, REFMULF and REFMUL3. REFMULF, is a 2D code able to cope with full polarization waves, treating all components of $\mathbf{E}=E_{x} \mathbf{e}_{\mathbf{x}}+E_{y} \mathbf{e}_{\mathbf{y}}+E_{z} \mathbf{e}_{\mathbf{z}}$ and $\mathbf{H}=H_{x} \mathbf{e}_{\mathbf{x}}+H_{y} \mathbf{e}_{\mathbf{y}}+H_{z} \mathbf{e}_{\mathbf{z}}$ of the wave. It supports a generic external magnetic field $\mathbf{B}_{\mathbf{0}}=B_{0_{x}} \mathbf{e}_{\mathbf{x}}+B_{0_{y}} \mathbf{e}_{\mathbf{y}}+B_{0_{z}} \mathbf{e}_{\mathbf{z}}$, coupling the Transverse-Electric Mode (TE, $X$-mode) with the Transverse-Magnetic Mode (TM, O-mode) via a linear vector differential equation for $\mathbf{J}$, which is solved using a modified Xu-Yuan kernel $[8,10]$, with extended long-run stability. The components of the external magnetic field $\mathbf{B}_{\mathbf{0}}$ lying on the propagation plane are responsible for linking the TE and TM modes, enabling the description of oblique propagation. For a $\mathbf{B}_{\mathbf{0}}$ purely perpendicular to the propagation plane, the code describes simultaneously O-mode and $\mathrm{X}$-mode propagation. This code enlarges the possibilities of simulation of microwave reflectometry offering capabilities unavailable in present day 2D reflectometry codes such as coupling O/X, Faraday rotation, and Cotton-Moutton effect, for instance. 

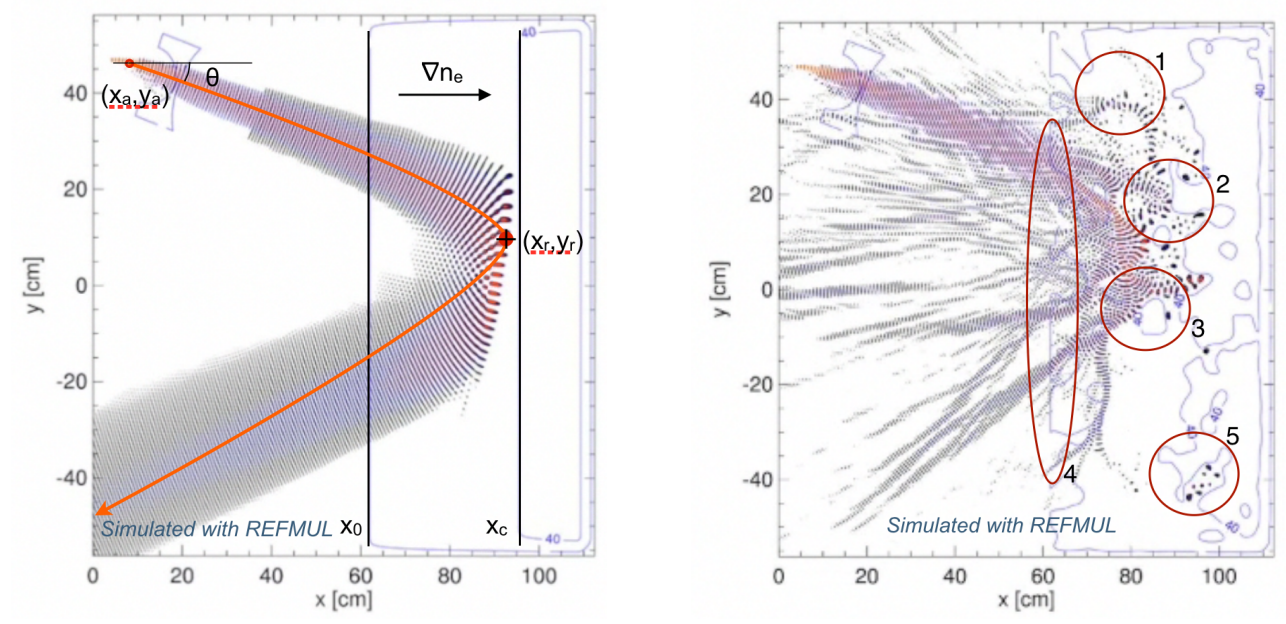

Figure 1. On the left, without turbulence, an analytical approach can still provide useful informations. With a turbulent plasma, on the right, the impact of forward scattering in a probing beam showing effects such as: 1-multi-scattering; 2-local resonances; 3-secondary cutoffs; 4-beam splitting and 5-wave trapping. These effects can only be assessed using a fullwave code.

REFMUL3, presently in an advanced stage of development, is a 3D full-wave code. Its numerical kernel shares a similar structure to REFMULF but involves more equations to be solved, since all field components are included in a volume. Furthermore, its 3D nature translates into increasing the grid-count by two or three orders of magnitude when compared with 2D codes, making the computational burden more stringent and the memory demands discouraging. This implied the usage of a parallel approach from the beginning, since only with a highly scalable parallel code can useful simulations be made. The REFMUL* family of codes aims primarily at improving the understanding of microwave reflectometry, which constitutes a standard diagnostic for current and future tokamaks. In this framework it has already been used to support physics studies in ASDEX Upgrade and Tore Supra. REFMUL has been used in the simulations for the design of Plasma-Position-Reflectometer for ITER [11] and also to assess its measurement behaviour [12]. This code was also used for the first assessment of microwave diagnostics for DEMO [13]. New simulations of DEMO to assess its PPRs' measurement performance are being made using REFMULF [14].

\subsection{REFMULF and REFMUL3 geometry and equations}

REFMULF, like the usual 2D O- and X-mode codes (such as REFMUL and REFMULX), also considers propagation in a 2D plane $(x-y$ plane) with no gradients along $z(\partial / \partial z=0)$. Maxwell equations are solved using the classic Yee schema on two sets of staggered grids, one for the transverse magnetic mode (TMz) with field components $E_{z}, H_{x}$ and $H_{y}$, and another for the transverse electric mode (TEz), with field components $H_{z}, E_{x}$ and $E_{y}$. The major difference from classic $2 \mathrm{D}$ codes is that a generic external magnetic field $\mathbf{B}_{\mathbf{0}}=B_{0_{x}} \mathbf{e}_{\mathbf{x}}+B_{0_{y}} \mathbf{e}_{\mathbf{y}}+B_{0_{z}} \mathbf{e}_{\mathbf{z}}$ is now allowed. The TMz and TEz planes are not coupled through Maxwell equations but coupling through the equation for $\mathbf{J}=J_{x} \mathbf{e}_{\mathbf{x}}+J_{y} \mathbf{e}_{\mathbf{y}}+J_{z} \mathbf{e}_{\mathbf{z}}$ is possible through the product $\boldsymbol{\omega}_{c} \times \mathbf{J}$, with $\boldsymbol{\omega}_{c}=\omega_{x} \mathbf{e}_{\mathbf{x}}+\omega_{y} \mathbf{e}_{\mathbf{y}}+\omega_{z} \mathbf{e}_{\mathbf{z}}$, being $\omega_{i}=\mathrm{e} B_{i} / m_{e}, i=x, y, z$. If the co-planar $\mathbf{B}_{\mathbf{0}_{\mathbf{x}}}\left(\omega_{x} \neq 0\right)$ and 

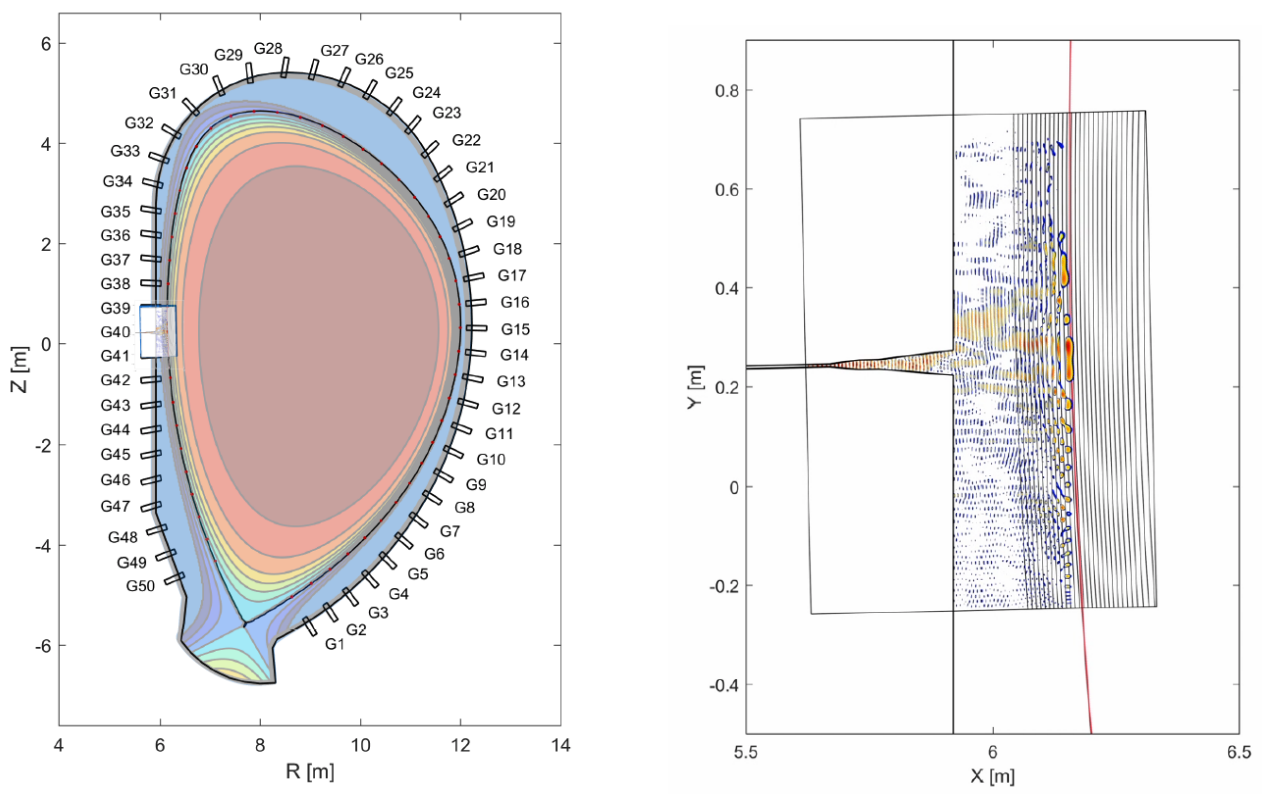

Figure 2. On the left a poloidal section of DEMO with a HFS equatorial reflectometer ROI is marked. A snapshot of the electric field in this ROI (G40), for a frequency of $f=43 \mathrm{GHz}$, appears on the right.

$\mathbf{B}_{\mathbf{0}_{\mathbf{y}}}\left(\omega_{y} \neq 0\right)$ are present, the TMz $\left(J_{z}\right)$ plane is coupled to TEz and the TEz $\left(J_{x}, J_{y}\right)$ plane to the TMz. For $\omega_{x}, \omega_{y}=0$, we revert to the uncoupled simultaneous description of O-mode and X-mode. REFMULF uses a variation of the Xu-Yuan schema [10] with the modifications proposed in [15] for extended long-run stability in time-dependent problems. A more detailed description of this schema can be found in [16].

REFMULF not far from a full polarization 3D code. In fact if we overlay $N$ TEz planes intermediated by $N-1 \mathrm{TMz}$ planes, instate dependency on $z$, e.g., $\mathbf{J}=\mathbf{J}(x, y, z)$, and allow for variation along the $z$ axis $(\partial / \partial z \neq 0)$, we obtain the bases of REFMUL3. For a more comprehensive description refer to [16]. One major difference while implementing REFMUL3 is that one must start thinking parallel. In order to have an usable 3D code we need a parallel kernel, parallel Input/Output (I/O) and parallel visualization of the results. This goal is achieved in REFMUL3 by employing a hybrid MPI/OpenMP parallelization with a 3D domain decomposition. This implementation shows a good strong scaling behaviour on the MARCONI cluster at Cineca Italy [16, 17].

\section{Examples of applications using REFMUL* codes}

REFMULF is being used to assess the performance of the future PPR of DEMO reactor and to evaluate how many views are necessary for a reconstruction of the plasma shape [14, 18]. In figure 2, on the left, a poloidal section of DEMO appears with several possible positions of the PPRs' access to the plasma. The rectangular region marks the region-of-interest (ROI) for a simulation of a reflectometer on the high field side at the equatorial plane. On the right, a snapshot of the electric field taken at a frequency of $f=43 \mathrm{GHz}$ is displayed for this ROI (G40). Multi-reflection structures can be clearly seen. The red line shows the position of the separatrix. 

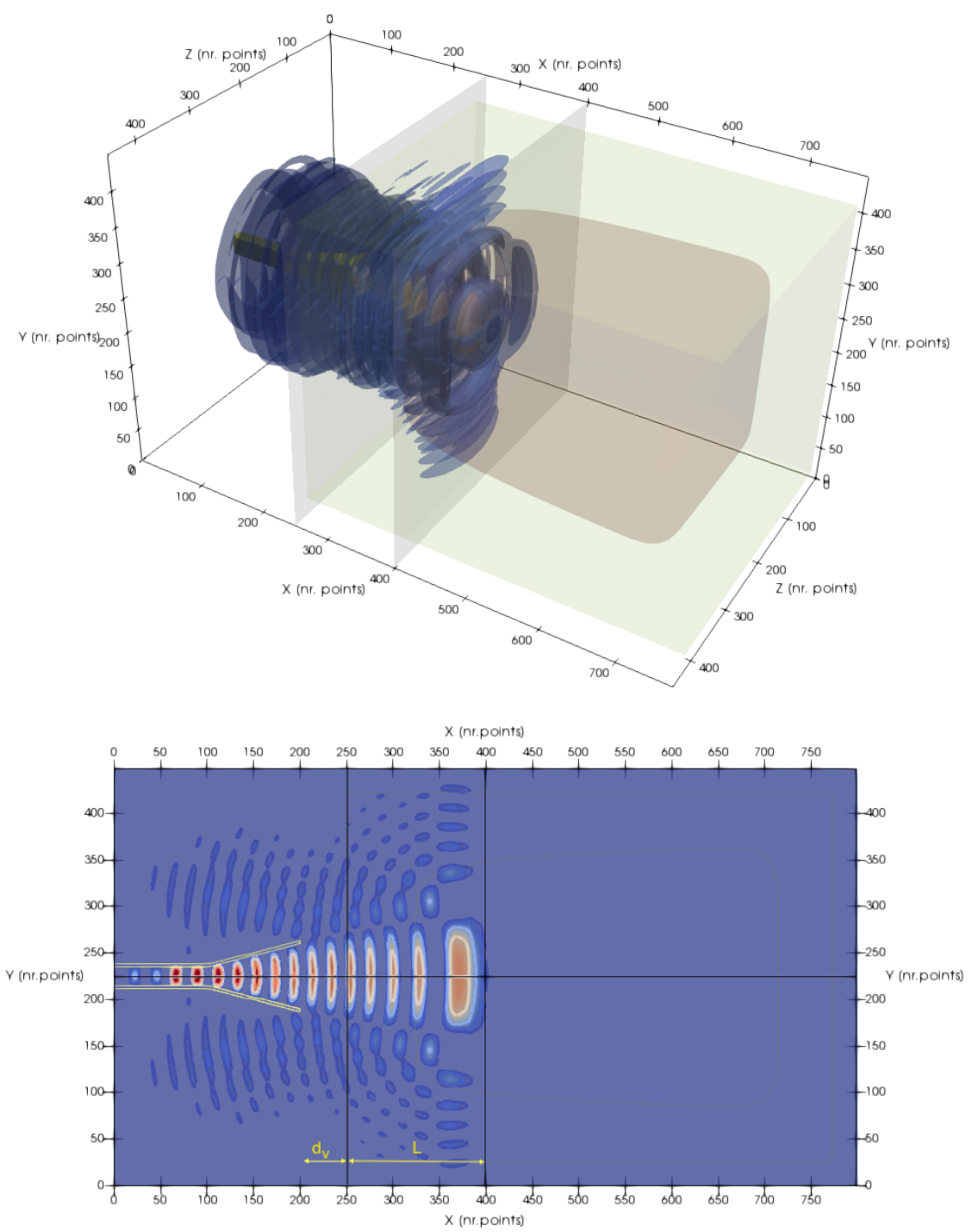

Figure 3. Parallel simulation of a plasma probing using a FFCW on a volume $800 \times 450 \times 450$. The 3D rendered volumes appear on the left while on the right a 2D contour of taken on the plane $x-y$ (antenna's H-plane).

As an example of the development status and usability of REFMUL3 we present the simulation of a plasma probing using O-mode fixed-frequency continuous-wave (FFCW) at a frequency $f=$ $30 \mathrm{GHz}$ probing a slab plasma with a distance from the edge to cutoff of $L=6.43 \lambda_{35 \mathrm{GHz}}$. The vacuum distance between the horn mouth and the plasma edge is $d_{v}=2.188 \lambda_{35 \mathrm{GHz}}$. A $3 \mathrm{D}$ Unidirectional Transparent Source (UTS) is implemented in the waveguide [8]. The waveguide and the standard horn are modelled as Perfect Electric Conductor (PEC). The simulation was performed on a volume of $800 \times 450 \times 450$ grid points with 8 MPI processes and 36 OpenMP threads each, for a total of 288 cores. In figure 3 , on the left, we visualise the 3D field structure of $E_{z}$ being reflected at the plasma and returning to the antenna. On the right, a contour of a cut done through the H-plane of the antenna ( $X-Y$ plane taken on the antenna's line of sight) is presented. 
Such simulations will improve the density profile reconstruction by giving a relevant evolution of the amplitude of the reflected wave and the effects of the open cavity one can find in the case of ITER PPRs.

\section{Conclusions}

Being numerical simulation of key importance to use reflectometry as a robust diagnostic for electronic density in plasmas, we have introduced the REFMUL* family of full-wave codes as a set tools devised for that purpose. These consist of several 2D codes, with different degrees of approximations, as well as a new 3D code. They allow to set up synthetic reflectometry diagnostics in order to assess their behaviour. An example of such a study was provided, in which an assessment of the Plasma Position Reflectometers of DEMO was made.

\section{Acknowledgments}

This work has been carried out within the framework of the EUROfusion Consortium and has received funding from the Euratom research and training programme 2014-2018 and 2019-2020 under grant agreement No 633053. The views and opinions expressed herein do not necessarily reflect those of the European Commission.

\section{References}

[1] C. Laviron, A.J.H. Donné, M.E. Manso and J. Sanchez, Reflectometry techniques for density profile measurements on fusion plasmas, Plasma Phys. Control. Fusion 38 (1996) 905.

[2] E. Mazzucato, Microwave reflectometry for magnetically confined plasmas, Rev. Sci. Instrum. 69 (1998) 2201.

[3] G. Breit and M.A. Tuve, A test of the existence of the conducting layer, Phys. Rev. 28 (1926) 554.

[4] H.I. Lassen, Über die Ionisation der Atmosphäre und ihren Einfluß auf die Ausbreitung der kurzen elektrischen Wellen der drahtlosen Telegraphie, Zeitschrift für Hochfrequenztechnik 28 (1926) 109.

[5] I.H. Hutchinson, Principles of Plasma Diagnostics, Cambridge University Press (1987).

[6] A. Taflove, Application of the finite-difference time-domain method to sinusoidal steady-state electromagnetic-penetration problems, IEEE Trans. Electromagn. Compat. 22 (1980) 191.

[7] K. Yee, Numerical solution of initial boundary value problems involving Maxwell's equations in isotropic media, IEEE Trans. Antennas Propagat. 14 (1966) 302.

[8] F. da Silva, S. Heuraux, S. Hacquin and M. Manso, Unidirectional transparent signal injection in finite-difference time-domain electromagnetic codes-application to reflectometry simulations, J. Comput. Phys. 203 (2005) 467.

[9] F. da Silva, S. Heuraux, B. Després and M. Campos Pinto, Numerical advances in kernels for FDTD Maxwell codes using the Yee algorithm, in proceedings of the $11^{\text {th }}$ International Reflectometry Workshop for fusion plasma diagnostics - IRW11, Palaiseau, France, 22-24 April 2013.

[10] L. Xu and N. Yuan, FDTD formulations for scattering from 3-d anisotropic magnetized plasma objects, IEEE Trans. Antennas Propagat. 5 (2006) 335. 
[11] S. Heuraux, R. Sabot, F. da Silva and G. Giruzzi, Final Report on Procurement Package 1, Diagnostic design for ITER-EFDA contract 06-1438, ITER-D-A6R2Z3 (2012).

[12] F. da Silva, S. Heuraux, E. Ricardo, P. Quental and J. Ferreira, Assessment of the measurement performance of the in-vessel systems of gaps 4 and 6 of the ITER Plasma Position Reflectometer using a FDTD Maxwell full-wave code, in proceedings of the $13^{\text {th }}$ International Reflectometry Workshop for fusion plasma diagnostics — IRW13, Daejeon, South Korea, 10-12 May 2017.

[13] A. G. Silva, F. da Silva, S. Heuraux and B. Gonçalves, First assessment of microwave diagnostics for DEMO, Fusion Eng. Des. 96-97 (2015) 948.

[14] E. Ricardo, F. da Silva, S. Heuraux and A. Silva, Assessment of the measurement performance of DEMO Plasma Position Reflectometry gaps perpendicular to the separatrix, presented at the $12^{\circ}$ Congresso do Comité Português da URSI "Inteligência artificial e as ciências rádio", Lisbon, Portugal, 14 December 2018.

[15] F. da Silva, M. Campos Pinto, B. Després and S. Heuraux, Stable explicit coupling of the yee scheme with a linear current model in fluctuating magnetized plasmas, J. Comput. Phys. 295 (2015) 24.

[16] F. da Silva, S. Heuraux and T. Ribeiro, Introducing REFMULF, a $2 D$ full polarization code and REFMUL3, a 3D parallel full wave Maxwell code, in proceedings of the $13^{\text {th }}$ International Reflectometry Workshop for fusion plasma diagnostics - IRW13, Daejeon, South Korea, 10-12 May 2017.

[17] R. Hatzky et al., HLST Core Team Report, EUROFUSION WPISA-REP(18) 20587 (2017).

[18] G. Marchiori, G. De Masi, R. Cavazzana, A. Cenedese, N. Marconato, R. Moutinho et al., Study of a plasma boundary reconstruction method based on reflectometric measurements for control purposes, IEEE Trans. Plasma Sci. 46 (2018) 1285. 